\title{
ZERO-PROFILE DEVICE IMPLANTATION IN ANTERIOR CERVICAL DIS- CECTOMY AND FUSION: A SINGLE INSTITUTE EXPERIENCE
}

\author{
Nattawut Niljianskul
}

Division of Neurosurgery, Department of Surgery, Faculty of Medicine Vajira Hospital, Navamindradhiraj University, Dusit, Bangkok 10300, Thailand

\begin{abstract}
Background: A surgical procedure, anterior cervical discectomy and fusion (ACDF), is used for neural decompression in degenerative cervical disk disease and cervical spondylosis. A new type of cervical interbody cage, the Zero-profile device (ZPD), has been developed which could reduce postoperative complications among patients.

Objectives: The study aimed to examine the effect of ZPD on clinical outcomes and cervical spine alignment of enrolled patients at 1-year follow-up in the management of ADCF.

Methods: This study retrospectively evaluated the clinical and radiographic outcomes using the Zero-profile device (ZPD) in the anterior cervical discectomy and fusion (ACDF). All patients who underwent ACDF at Vajira Hospital between May 2017 and June 2021 were included in this study. Radiographic images obtained from picture archiving and communication systems (PACS) were used to evaluate the device-level Cobb angle (DLCA), segmental Cobb angle (SCA), global Cobb angle (GCA), sagittal vertical axis (SVA), and intervertebral disk height. The Japanese Orthopaedic Association (JOA) scores and visual analog scale (VAS) were obtained from the patients' medical records. The preoperative DLCA, SCA, GCA, SVA, and intervertebral disk height measurements were compared with the postoperative measurements at 1 year.

Results: A total of 31 patients (45 disks) who underwent ACDF with the ZPD were included in this study. A significant improvement was found in JOA, VAS, DLCA, SCA, GCA, SVA, and intervertebral disk height after ACDF with ZPD $(p<0.001)$. Immediate postoperative dysphagia occurred in two patients $(6.5 \%)$, which resolved after 3 months. No subsidence was reported at 1-year follow-up. Age, BMI as well as the preoperative cervical alignment did not affect outcomes in this study.

Conclusion: The use of the ZPD in ACDF improved clinical and radiographic outcomes in the correction of cervical spine alignment, and minimized postoperative complications of dysphagia. No device-related failure occurred, and favorable outcomes persisted at 1-year follow-up.
\end{abstract}

Keywords: Alignment, Anterior cervical discectomy and fusion, Zero-profile device

J Southeast Asian Med Res 2022; 6: e0104

https://doi.org/10.55374/jseamed.v6i0.104

Correspondence to:

Niljianskul N, Division of Neurosurgery, Department of Surgery, Faculty of Medicine Vajira Hospital, Navamindradhiraj University, Dusit, Bangkok 10300, Thailand

E-mail: nattawut@nmu.ac.th

Received: 1 September 2021

Revised: 22 November 2021

Accepted: 3 December 2021 


\section{Introduction}

Anterior cervical discectomy and fusion (ACDF) is a surgical procedure for neural decompression in degenerative cervical disk disease and cervical spondylosis and is performed using an anterior approach. After the discectomy, the surgeon inserts an autologous bone graft or traditional interbody cage and plate structure to restore the height of the intervertebral space, reconstructs the cervical spine curvature and maintains cervical spine stability. Reported complications of this procedure include dysphagia, odynophagia and hoarseness. Further, cage subsidence can lead to implant failure or adjacent segment pathology. A new type of cervical interbody cage, the Zero-profile device (ZPD), has been developed. The ZPD comprises an interbody polyetheretherketone (PEEK) cage and an internal implant with locking screws. The characteristics of PEEK are well suited to ACDF because it has elastic properties that closely match cortical bone. ${ }^{(1)}$ The ZPD cervical interbody cage is held with screws inserted into the adjacent vertebral bodies, fixing the implant in the intervertebral disc space after discectomy. No cervical plate is attached between the anterior surface of the vertebral body and esophagus or any prevertebral soft tissue with the ZPD, which may lead to a reduction in postoperative dysphagia. (2)

Furthermore, the shape of the ZPD is lordotic and is similar to the normal anatomical alignment of the cervical spine. Many studies have indicated that cervical kyphosis alone without cord compression increases longitudinal cord tension and intramedullary pressure. This has been shown to cause neuronal loss and demyelination in animal models. ${ }^{(3,4)}$ The loss of lordosis and the development of kyphosis can cause neurological deterioration and should be avoided. ${ }^{(5)}$ Koeppen et al. reported a study of 102 patients with cervical spondylosis myelopathy involving 219 fused levels with a traditional lordotic PEEK cage. They demonstrated that kyphosis was associated with pre- and postoperative neck pain. ${ }^{(6)}$ The hypothesis of this study constituted whether the ZPD may improve cervical sagittal alignment. Thus, this study aimed to examine the effect of the ZPD on clinical outcomes and cervical spine alignment of enrolled patients at 1-year follow-up in the management of ADCF.

\section{Methods}

This study was approved by the Ethics Committee of Vajira Hospital (045/64). All patients who undergoing ACDF at the Neurosurgical Department of Vajira Hospital between May 2017 and June 2021 were enrolled. The indications for ACDF were cervical spine injuries, cervical spondylosis, cervical spondylodiscitis, and ossification of the posterior longitudinal ligament of the cervical spine. Patients with no record of the cage type in the operation notes, with no pre- or postoperative imaging, or incomplete follow-up data were excluded. A total of 31 patients undergoing ACDF with the ZPD implantation in 45 disk levels were included in this study.

The pre-operative visual analog scale (VAS) for neck pain and Japanese Orthopaedic Association (JOA) score were assessed, and a cervical spine $\mathrm{X}$-ray and spine MRI were performed. General anesthesia was performed without neuromuscular blockers or agents that affected neuromuscular monitoring. Intra-operative neuromuscular monitoring was performed to evaluate somatosensory-evoked potentials (SSEPs) and transcranial motor-evoked potentials (tcMEP). Electromyography (EMG) was also performed. A train of four twitches was used at the common peroneal nerve, and a response rate $\geq 75 \%$ was required before recording the EMG.

Among all patients, surgery was performed by an anterior cervical approach using the SmithRobinson technique. The surgeons comprised two experienced spinal neurosurgeons with more than five years' experience and a cervical retractor was applied for distraction. Discectomy, removal of osteophytes with a high speed drill and Kerrison rongeurs and opening of the posterior longitudinal ligament was performed in all cases under a microscope. After preparation of the fusion bed, interbody fusion was performed with the ZPD. All the ZPDs, filled with bone graft substitute, were $17 \mathrm{~mm}$ wide, $14 \mathrm{~mm}$ long, and exhibited a $7^{\circ}$ lordotic taper. Multiple implant heights 
accommodated the varied patient anatomy. Robust implants with 3 screws, $4 \mathrm{~mm}$ in diameter, were inserted using a freehand technique. The angled instruments were designed to work perpendicular to the spine. The correct screw angle and trajectory were automatically achieved when the screwdrivers were seated properly within the screw hole. The length of the screws was measured from the anterior portion of the implant to the total distance reached posteriorly. The trajectory of the screws was at about a 40-degree angle to the superior and inferior surfaces of the ZPD. A soft collar was applied for six weeks after surgery among all patients.

\section{Postoperative evaluation and radiographic outcomes}

All patients underwent a physical examination and radiography of the cervical spine at 1, 3, 6 and 12 months after surgery. The X-rays were reviewed, and the operator and another neurosurgeon measured the parameters using a single measurement before and after surgery at 12 months using picture archiving and communication systems (PACS) measurement features. The sagittal alignment was assessed using a device-level Cobb angle (DLCA) at each operative level, segmental Cobb angle (SCA), global Cobb angle (GCA) and C2 to 7 sagittal vertical axis (SVA). Pre- and postoperative imaging at one year were compared. DLCA was measured by drawing lines parallel to the inferior endplate of the upper

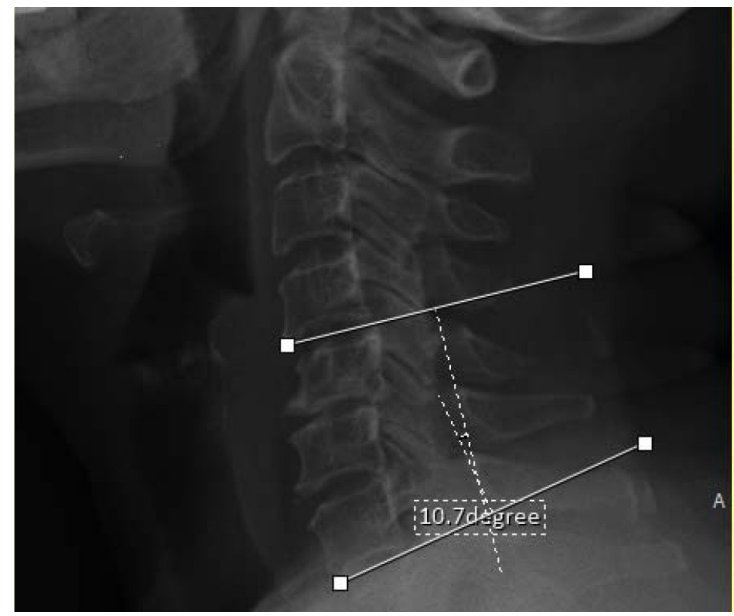

operative vertebral level and the inferior endplate of the lower operative vertebral level. SCA was measured by drawing lines parallel to the inferior endplate of the uppermost operative vertebral level and the inferior endplate of the lowermost operative vertebral level. Perpendicular lines were then drawn from each of the above two lines, and the angle of intersection constituted the SCA(Figure 1). GCA was measured by drawing lines parallel to the inferior endplate of $\mathrm{C} 2$ and the inferior endplate of C7. Perpendicular lines were drawn from each of the lines, and the angle of intersection was the GCA (Figure 2). C2 to 7 SVA comprised the distance in $\mathrm{mm}$ measured between a plumb line dropped from the centroid of $\mathrm{C} 2$, and another plumb line dropped from the posterosuperior aspect of the C7 vertebral body (Figure 3). Pre- and postoperative (at one-year follow-up) ventral and reduced dorsal segmental height were measured and compared. A height reduction of more than $3 \mathrm{~mm}$ ventrally or dorsally was defined as subsidence. ${ }^{(7)}$ Fusion was primarily assessed using cervical X-rays and bridging trabecular bone between the endplates. The absence of a radiolucent gap between the endplate and graft was evidence for osseous union. When it remained unclear, flexion and extension cervical views showing $<1 \mathrm{~mm}$ of motion between the spinous processes confirmed fusion. Operative time, blood loss, and complications were recorded. Dysphagia was evaluated according to the Bazaz grading system. ${ }^{(8)}$

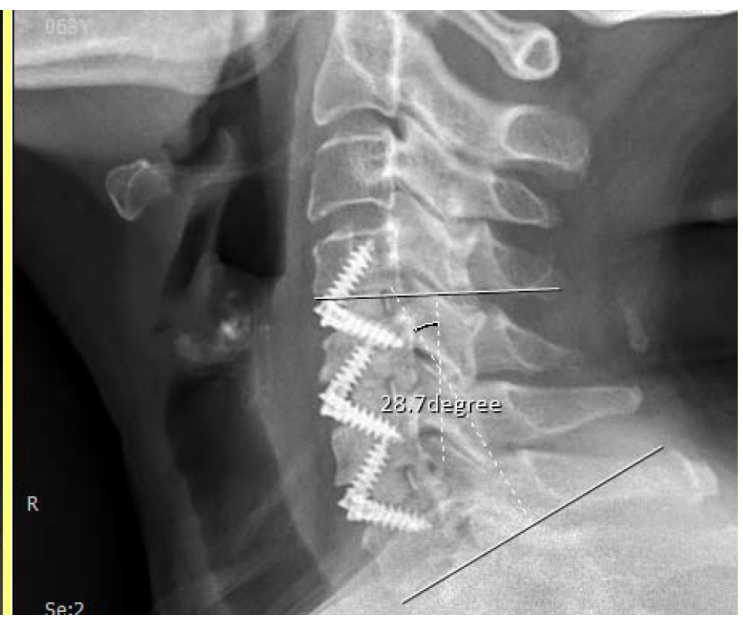

Figure 1. Segmental Cobb angle (SCA) was measured by drawing lines parallel to the inferior endplate of the uppermost operative vertebral level and the inferior endplate of the lowermost operative vertebral level. Perpendicular lines were then drawn from each of the above two lines, and the angle of intersection constituted the SCA. Pre- and postoperative imaging at one year were compared. 

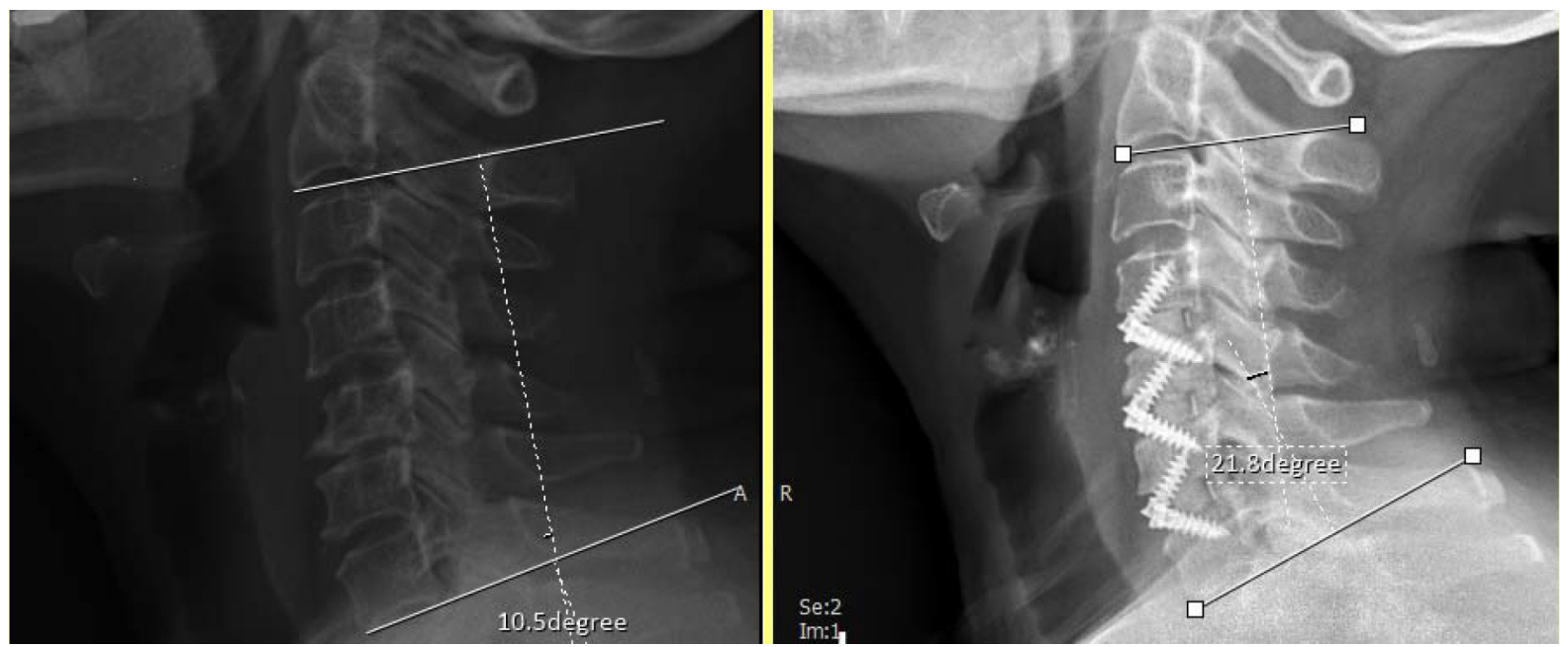

Figure 2. GCA was measured by drawing lines parallel to the inferior endplate of $\mathrm{C} 2$ and the inferior endplate of C7. Perpendicular lines were drawn from each of the lines, and the angle of intersection comprised the GCA. Pre- and postoperative imaging at one year were compared.
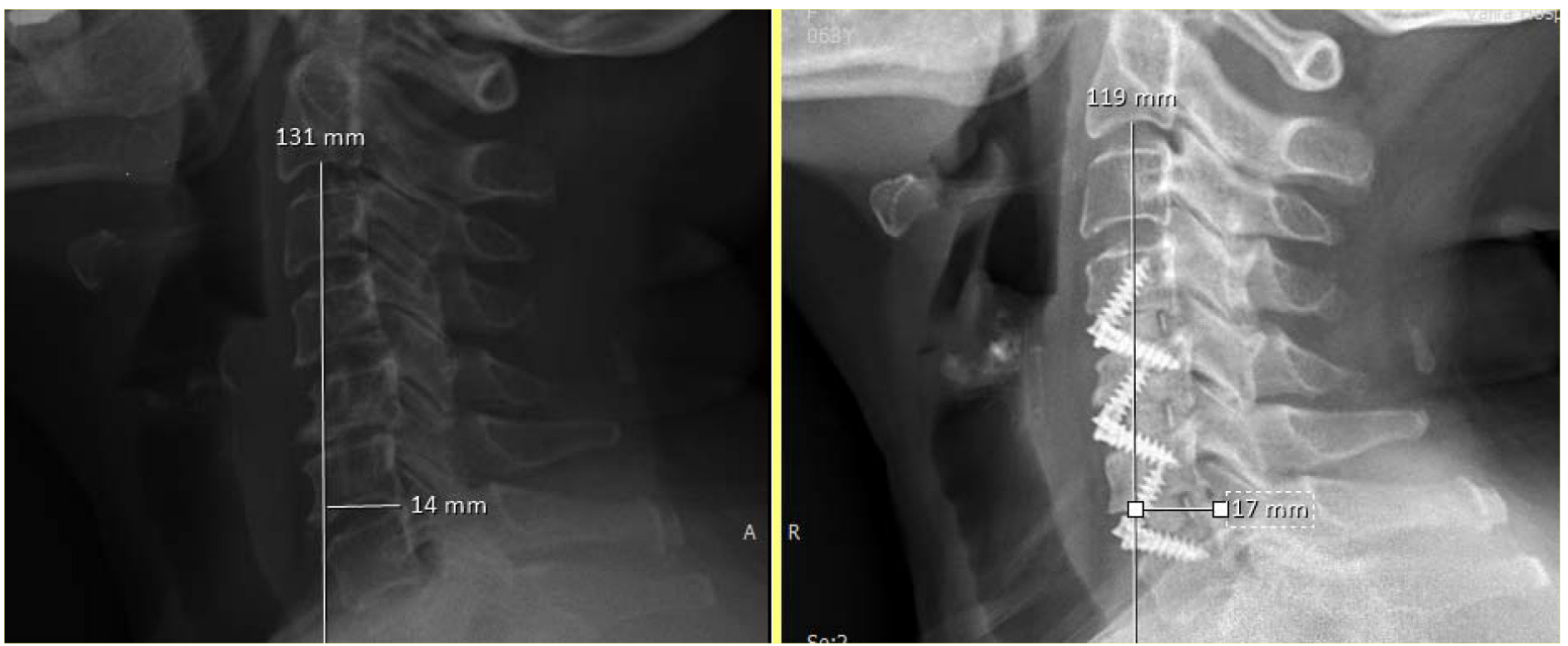

Figure 3. $\mathrm{C} 2$ to $7 \mathrm{SVA}$ is the distance in $\mathrm{mm}$ measured between a plumb line dropped from the center of $\mathrm{C} 2$, and another plumb line dropped from the posterosuperior aspect of the $\mathrm{C} 7$ vertebral body. Pre- and postoperative imaging at one year were compared.

\section{Statistical analysis}

Statistical analysis was performed using the Statistical Package for the Social Sciences Software, Version 22 for Windows (SPSS Inc., Chicago, IL, USA). The t-test was used to analyze continuous quantitative variables. Pre-and postoperative cervical parameters were compared by ANOVA and to calculate the p-value. A $p$-value $<0.050$ was considered statistically significant.

\section{Results}

\section{Patient and treatment characteristics}

In total, 31 patients were initially included, and 45 fused intervertebral discs were analyzed.
The demographic data of the patients are presented in Table 1. Of these, 17 males and 14 females had a median age of 57 ( 25 to 84 ) years. The most common indications for ACDF were spondylosis $(64.5 \%)$ and trauma $(32.3 \%)$. The preoperative curvature of the cervical spine was kyphosis among 12 patients $(38.7 \%)$ and lordosis among 19 patients (61.3\%). Most patients underwent single level ACDF (61.3\%), and the most common fused level was C5/6 (67.7\%). The mean operative time was 202 minutes, and the mean operative blood loss was $96 \mathrm{~mL}$. No patients had pre-existing dysphagia or gastro-esophageal reflux disease before surgery. No postoperative neurological 
deficits, infections, or hematomas were observed. Immediate postoperative dysphagia occurred in two patients $(6.5 \%)$, which resolved within three months. None of the patients presented postoperative dysphagia beyond three months.

Table 1. Patient and treatment characteristic
The two cases that had immediate postoperative dysphagia had been operated on at level $\mathrm{C} 5 / 6$ and $\mathrm{C} 6 / 7$ and had a BMI $>27.5 \mathrm{~kg} / \mathrm{m} 2$. No cage subsidence was found at one-year follow-up.

\begin{tabular}{|c|c|}
\hline Characteristic & Patients $(n=31)$ \\
\hline \multicolumn{2}{|l|}{ Sex } \\
\hline Male & $17(54.8)$ \\
\hline Female & $14(45.2)$ \\
\hline Age, years & $57(26.0-84.0)$ \\
\hline \multicolumn{2}{|l|}{ Diagnosis } \\
\hline Trauma & $10(32.3)$ \\
\hline Spondylosis & $20(64.5)$ \\
\hline Infection & $1(3.2)$ \\
\hline \multicolumn{2}{|l|}{ Curvature of cervical spine } \\
\hline Lordosis & $19(61.3)$ \\
\hline Kyphosis & $12(38.7)$ \\
\hline Operation time (minutes) & $202(95.0-480.0)$ \\
\hline Blood loss (milliliter) & $96(10.0-680.0)$ \\
\hline Length of stay (day) & $8(3.0-25.0)$ \\
\hline \multicolumn{2}{|l|}{ Fused level } \\
\hline $\mathrm{C} 3 / 4$ & $6(19.4)$ \\
\hline $\mathrm{C} 4 / 5$ & $8(25.8)$ \\
\hline $\mathrm{C} 5 / 6$ & $21(67.7)$ \\
\hline $\mathrm{C} 6 / 7$ & $10(32.3)$ \\
\hline \multicolumn{2}{|l|}{ Number of fused level } \\
\hline 1 & $19(61.3)$ \\
\hline 2 & $10(32.3)$ \\
\hline 3 & $2(6.5)$ \\
\hline \multicolumn{2}{|l|}{ Post-operative complications } \\
\hline Dysphagia & $2(6.5)$ \\
\hline Hoarseness & $2(6.5)$ \\
\hline None & $27(87.1)$ \\
\hline
\end{tabular}

Values are presented as number (\%) or median (range) 
Table 2. Comparison of clinical and radiographic features

\begin{tabular}{lcccc}
\hline Variable & $\begin{array}{c}\text { Pre- } \\
\text { operative }\end{array}$ & $\begin{array}{c}\text { Post- } \\
\text { operative }\end{array}$ & Change & $\boldsymbol{p}$-value \\
\hline VAS neck & $5.8(1.5)$ & $0(0.3)$ & $5.7(1.5)$ & $<0.001$ \\
JOA & $3.7(2.6)$ & $8.8(4.4)$ & $5.1(4.2)$ & $<0.001$ \\
DLCA, degree & $12.6(3.6)$ & $15.1(3.3)$ & $2.5(1.3)$ & $<0.001$ \\
SCA, degree & $4.8(3.4)$ & $10.9(4.8)$ & $6.1(4.3)$ & $<0.001$ \\
GCA, degree & $11.8(7.6)$ & $18.1(6.8)$ & $6.3(7.1)$ & $<0.001$ \\
C2-7 SVA, cm & $1.8(1.1)$ & $2.7(1.1)$ & $0.9(1.3)$ & $<0.001$ \\
Intervertebral disc height, cm & $4.0(1.4)$ & $4.9(1.7)$ & $0.9(0.7)$ & $<0.001$ \\
\hline
\end{tabular}

Values are presented as mean (SD)

$\mathrm{VAS}=$ Visual analogue scale for neck pain, JOA=the Japanese Orthopaedic Association score,

$\mathrm{DLCA}=$ device level Cobb angle, $\mathrm{SCA}=$ segmental $\mathrm{Cobb}$ angle, $\mathrm{GCA}=$ global Cobb angle, $\mathrm{SVA}=$ sagittal vertical axis, $\mathrm{cm}=$ centimeter

Table 3. Subgroup analysis by change of pre-operative alignment

\begin{tabular}{lccccc}
\hline \multirow{1}{*}{ Variable } & \multicolumn{2}{c}{ Initial lordosis } & \multicolumn{2}{c}{ Initial kyphosis } & \multirow{2}{*}{-value } \\
\cline { 2 - 5 } & Means (SD) & No. & Means (SD) & No. & \\
\hline$\Delta$ DLCA, degree & $5.8(4.1)$ & 15 & $4.7(4.1)$ & 30 & 0.424 \\
$\Delta$ SCA, degree & $6.0(4.3)$ & 11 & $6.1(4.3)$ & 20 & 0.911 \\
$\Delta$ GCA, degree & $3.0(5.8)$ & 11 & $8.1(7.3)$ & 20 & 0.055 \\
$\Delta$ C2-7 SVA, cm & $2.6(1.4)$ & 11 & $0.8(1.2)$ & 20 & 0.430 \\
$\begin{array}{lllll}\Delta \text { Intervertebral disc } \\
\text { height, cm }\end{array}$ & $0.9(0.6)$ & 11 & $1.0(0.7)$ & 20 & 0.637 \\
\hline
\end{tabular}

Values are presented as mean (SD).

Abbreviations: $\Delta=$ change in, $\mathrm{DLCA}=$ device level Cobb angle, SCA=segmental Cobb angle, $\mathrm{GCA}=$ global Cobb angle, $\mathrm{SVA}=$ sagittal vertical axis, $\mathrm{cm}=$ centimeter

\section{Comparison of clinical and radiographic features}

The mean changes for patients undergoing ACDF with the ZPD were documented. The mean VAS for neck pain across the cohort was $5.8( \pm 1.5)$ pre-operatively and $0( \pm 0.3)$ postoperatively, improving by a mean of $5.7( \pm 1.5)$, which was considered statistically significant. The mean JOA across the cohort was $12.6( \pm 3.6)$ pre-operatively and $15.1( \pm 3.3)$ postoperatively, improving by a statistically significant mean of $2.5( \pm 1.3)$.
The mean parameters including DLCA, SGA GCA and SVA improved toward lordosis after surgery, which were considered statistically significant. The mean fused level intervertebral disk height was $4.0( \pm 1.4 \mathrm{~mm})$ pre-operatively and $4.9( \pm 1.7 \mathrm{~mm})$ at one-year follow-up, demonstrating a significant increase between pre-operative and postoperative measurements (Table 2). 
Table 4. Subgroup analysis by change of age

\begin{tabular}{lccccc}
\hline \multirow{1}{*}{ Variable } & \multicolumn{2}{c}{ Age $\leq \mathbf{6 0}$} & \multicolumn{2}{c}{ Age $>\mathbf{6 0}$} & \multirow{2}{*}{-value } \\
\cline { 2 - 5 } & Means (SD) & No. & Means (SD) & No. & \\
\hline$\Delta$ DLCA, degree & $5.4(4.6)$ & 27 & $4.7(3.7)$ & 19 & 0.543 \\
$\Delta$ SCA, degree & $5.7(4.5)$ & 20 & $6.8(3.8)$ & 11 & 0.429 \\
$\Delta$ GCA, degree & $6.7(8.1)$ & 20 & $5.7(8.1)$ & 11 & 0.984 \\
$\Delta$ C2-7 SVA, cm & $0.7(1.0)$ & 20 & $1.1(1.6)$ & 11 & 0.598 \\
$\begin{array}{l}\text { Intervertebral disc } \\
\text { height, cm }\end{array}$ & $0.9(0.7)$ & 20 & $1.0(0.7)$ & 11 & 0.329 \\
\hline
\end{tabular}

Values are presented as mean (SD).

Abbreviations: $\Delta=$ change in, $\mathrm{DLCA}=$ device level Cobb angle, $\mathrm{SCA}=$ segmental Cobb angle, $\mathrm{GCA}=$ global Cobb angle, $\mathrm{SVA}=$ sagittal vertical axis, $\mathrm{cm}=$ centimeter

Table 5. Subgroup analysis by change BMI

\begin{tabular}{|c|c|c|c|c|c|}
\hline \multirow{2}{*}{ Variable } & \multicolumn{2}{|c|}{ BMI $(<27.5)$} & \multicolumn{2}{|c|}{ BMI (> 27.5) } & \multirow{2}{*}{$p$-value } \\
\hline & Change & No. & Change & No. & \\
\hline$\triangle \mathrm{DLCA}$, degree & $5.2(4.2)$ & 34 & $4.1(3.6)$ & 11 & 0.439 \\
\hline$\Delta \mathrm{SCA}$, degree & $6.2(4.4)$ & 23 & $6.6(3.8)$ & 8 & 0.839 \\
\hline$\Delta \mathrm{GCA}$, degree & $7.2(6.4)$ & 23 & $5.4(5.5)$ & 8 & 0.119 \\
\hline$\Delta \mathrm{C} 2-7 \mathrm{SVA}, \mathrm{cm}$ & $1.0(1.3)$ & 23 & $0.8(1.2)$ & 8 & 0.718 \\
\hline $\begin{array}{l}\Delta \text { Intervertebral disc } \\
\text { height, } \mathrm{cm}\end{array}$ & $0.9(0.7)$ & 23 & $1.1(0.8)$ & 8 & 0.390 \\
\hline
\end{tabular}

Values are presented as mean (SD).

Abbreviations: $\Delta=$ change in, $\mathrm{DLCA}=$ device level Cobb angle, $\mathrm{SCA}=$ segmental Cobb angle, $\mathrm{GCA}=$ global Cobb angle, $\mathrm{SVA}=$ sagittal vertical axis, $\mathrm{cm}=$ centimeter

Table 6. Subgroup analysis by change of level of discectomy

\begin{tabular}{|c|c|c|c|c|c|}
\hline \multirow{2}{*}{ Variable } & \multicolumn{2}{|c|}{1} & \multicolumn{2}{|c|}{ More than 1} & \multirow{2}{*}{$p$-value } \\
\hline & Change & No. & Change & No. & \\
\hline$\Delta \mathrm{DLCA}$, degree & $4.6(2.2)$ & 19 & $3.2(2.7)$ & 26 & 0.391 \\
\hline$\Delta \mathrm{SCA}$, degree & $5.7(4.6)$ & 19 & $6.6(3.8)$ & 12 & 0.429 \\
\hline$\Delta \mathrm{GCA}$, degree & $6.9(8.1)$ & 19 & $5.4(5.5)$ & 12 & 0.984 \\
\hline$\Delta \mathrm{C} 2-7 \mathrm{SVA}, \mathrm{cm}$ & $1.0(1.3)$ & 19 & $0.8(1.2)$ & 12 & 0.598 \\
\hline $\begin{array}{l}\Delta \text { Intervertebral disc } \\
\text { height, } \mathrm{cm}\end{array}$ & $0.8(0.6)$ & 19 & $1.1(0.8)$ & 12 & 0.329 \\
\hline
\end{tabular}

Values are presented as mean (SD).

Abbreviations: $\Delta=$ change in, DLCA=device level Cobb angle, $\mathrm{SCA}=$ segmental Cobb angle, $\mathrm{GCA}=$ global Cobb angle, $\mathrm{SVA}=$ sagittal vertical axis, $\mathrm{cm}=$ centimeter 
The results were analyzed based on preoperative alignment in which 11 patients had cervical lordosis, and 20 had cervical kyphosis. In patients with any cervical alignment, the SA parameters were improved toward lordosis, but was not statistically significant (Table 3). In Table 4, the JOA score and the changes in DLCA, SCA, GCA, SVA, and fused level intervertebral disk height were compared between patients older than 60 years and those 60 years and younger. No statistically significant differences were found between the two age groups regarding the clinical outcome, cervical alignment parameters, and fused level intervertebral disk height for patients undergoing ACDF with lordotic PEEK cages.

Table 5 shows the subgroup analysis by BMI. Every subgroup of BMI exhibited improving JOA score, changes in DLCA, SCA, GCA, SVA, and fused level intervertebral disk height but they were not statistically significance. Single or multilevel ACDF indicated no statistically significant difference in clinical or radiographic outcomes (Table 6).

\section{Discussion}

According to in vitro biomechanical studies, the ZPD provides comparable biomechanical stability to that of the traditional interbody cage and plate structure. ${ }^{(9,10)}$ Clinical studies have also indicated that the ZPD is safe and efficient, even in multilevel cases. ${ }^{(11,12)}$ Cervical spine alignment is one of the most important influences on clinical outcomes. According to a meta-analysis, ACDF with ZPD significantly improved postoperative GCA and curvature of the cervical spine. It has been reported that a single-level ACDF might not substantially impact cervical sagittal alignment. ${ }^{(13)}$ This study found no statistically significant differences between clinical or radiographic outcomes for single or multilevel ACDF (Table 6). Patients presenting single-level ACDF may increase lordosis due to lordotic PEEK cage and obtain better posture after surgery. For multilevel ACDF, even more than one cage has been implanted; however, postoperative lordosis changes are likely to decrease due to stiffness of neck in a long term.
In contrast, with previous cohorts, the author found that ACDF with ZPD, regardless of the number of discectomy levels, could significantly improve sagittal alignment. This study also found that ACDF with ZPD improved the JOA score and demonstrated a statistically significant restoration of cervical lordosis alignment. Furthermore, among patients with pre-operative cervical kyphosis or lordosis, the use of a ZPD significantly increased the sagittal alignment parameters in both groups. The author considers that patients who had any alignment of the preoperative cervical curve were likely to benefit from using the ZPD during ADCF. The shape of the ZPD is $7^{\circ}$ lordotic, which may restore the lordotic neck curvature of these patients. In our study, all parameters of sagittal alignment were improved toward lordosis after surgery.

ACDF is a common operative treatment for cervical disk pathology. It has been generally accepted that using an anterior cervical plate construct after interbody cage implantation promotes successful fusion. ${ }^{(14-16)}$ However, this procedure can result in various complications. The overall reported incidence of dysphagia after anterior cervical spine surgery varied from 2 to $60 \%{ }^{(17-19)}$ Furthermore, according to many studies, ACDF with an anterior cervical plate can lead to postoperative dysphagia in 2.0 to $67.0 \%$ of patients in the early postoperative period. ${ }^{(8)}$ Most of these symptoms disappear within three months of surgery. The incidence of chronic dysphagia after ACDF is between 3.0 and $21.0 \%$. ${ }^{(20)}$ The pathologic mechanism of postoperative dysphagia remains unknown, but may be associated with direct impingement of the implant on the posterior esophagus. ${ }^{(21)}$ Only two patients $(6.5 \%)$ reported postoperative dysphagia in this cohort, which resolved within three months. The author considers that ACDF with ZPD could minimize the postoperative dysphagia. The ZPD has advantages in this regard because it can be implanted completely inside the decompressed intervertebral disc space and fixed with integrated screws without an anterior cervical plate which could irritate the esophagus.

According to many studies, the age and BMI of patients may affect the outcome of ACDF. Di Capua et al. retrospectively investigated 20,563 
patients between 2010 and 2014. They found that elderly patients 61 years old and over had an increased risk of pulmonary and cardiac complications, venous thromboembolism, infection, and unplanned readmission. ${ }^{(22)}$ Omidi et al. demonstrated that ACDF is more effective at improving disability among patients over 45 years of age. ${ }^{(23)}$ Chotai et al. reported that patients older than 65 years have a slightly higher cost use ratio than younger patient groups, and surgery in the older cohort had significantly improved pain, disability, and quality of life outcomes. ${ }^{(24)}$ Obesity is an increasing global public health issue. The prevalence of obesity (BMI $\geq 25 \mathrm{~kg} / \mathrm{m}^{2}$ ) among adults in Thailand increased from 13.0\% among men and 23.2\% among women in 1991 to 22.4 and $34.3 \%$, respectively, in 2004. ${ }^{(25)}$ Obese patients had a greater potential for complications such as intra-operative durotomy, dysphagia, neurological, cardiopulmonary, and hematologic complications following ACDF procedures. ${ }^{(26)}$ Basques et al. reported that obesity did not impact clinical outcomes but affected cervical sagittal alignment and adjacent segment degeneration in a short term follow-up after ACDF. ${ }^{(27)}$ In the current study, although the two cases with postoperative dysphagia were classified as obese, after multivariate analysis, the author found the relationship without statistical significance. Furthermore, the current study found no age-related effects in the clinical outcomes, complications, cervical sagittal alignment parameters, or the fused level intervertebral disk height in ACDF with the ZPD. This indicated that patients can benefit from the ZPD regardless of age and BMI. Age is a nonmodifiable risk factor; and therefore, presents a fixed source of postoperative risk following elective ACDF. The author considers that the outcome of ACDF may be improved by intraoperative neuromonitoring, the use of a microscope during surgery, and the influence of experienced surgeons. This issue may be worth further study.

Cage subsidence is common after ACDF and can lead to deterioration of long term function. It causes loss of the correction of the segmental angle and the Cobb angle from $\mathrm{C} 2-7$. Criteria for evaluating subsidence remain insufficient.
The most common method is to measure the postoperative reduction in the heights of the ventral and dorsal segments between the two fused vertebral bodies. A decrease in total intervertebral disc height of $2 \mathrm{~mm}$ or more is significant subsi dence. Subsidence of less than $2 \mathrm{~mm}$ is acceptable. The incidence of cage subsidence with interbody PEEK cages ranges from 0.0 to $18.0 \%$ in the literature. ${ }^{(5,28)}$ Lee et al. reported that the subsidence rate of a ZPD (58.6\%) was higher than that of an interbody PEEK cage with plate (38.5\%), indicating that plate fixation can prevent subsidence by supporting the anterior disk height. ${ }^{(29)}$ In contrast, Scholz et al. reported that cage subsidence was not observed in cases with a ZPD, although their follow-up was only six months. ${ }^{(30)}$ In this cohort, no subsidence was reported at one-year follow-up. The reason for this difference may be that performing ACDF with a plate involves flattening the anterior vertebral surface of the cervical spine by resecting osteophytes to enable the plate to fit closely. Inserting the ZPD does not require this, and in addition to a secure and rigid screw fixation, it probably contributes to preserving the anterior bony support and reducing subsidence. The author considers that using the ZPD in ACDF may not result in subsidence after surgery; however, a larger sample size and longer follow-up period are needed to confirm this. The occurrence of cage subsidence may be related to several factors, including the size and position of the cage, bone density, and the contact surface ratio of the cage.

Several limitations, encountered in this study, need to be acknowledged. It constituted a retrospective study that did not compare the ZPD with other types of cervical cage. Furthermore, the length of follow-up was short so that the incidence of late complications such as subsidence, adjacent segment pathology, or device-related failure could not be assessed. In addition, the variation of diseases in this study was quite diverse. The vertebral body might not have responded best regarding the trauma and infection and could have affected the result analysis. Moreover, only a small number of available patients from a single institute were included. 


\section{Conclusion}

The use of the ZPD in ACDF surgery improved both clinical and radiographic outcomes by correcting cervical spine alignment and minimizing the postoperative complication of dysphagia. No device-related failure was observed, and favorable outcomes persisted at one-year follow-up. Age, BMI, and pre-operative cervical alignment did not affect outcomes in this study.

\section{Conflicts of Interest}

The author has no conflicts of interest to declare.

\section{References}

1. Boakye M, Mummaneni PV, Garrett M, Rodts G, Haid R. Anterior cervical discectomy and fusion involving a polyetheretherketone spacer and bone morphogenetic protein. $\mathrm{J}$ Neurosurg Spine 2005; 2: 521-5.

2. Vanek P, Bradac O, Delacy P, Lacman J, Benes V. Anterior interbody fusion of the cervical spine with Zero-P spacer: prospective comparative study-clinical and radiological results at a minimum 2 years after surgery. Spine (Phila Pa 1976) 2013; 38: E792-7.

3. Farley CW, Curt BA, Pettigrew DB, Holtz JR, Dollin N, Kuntz C 4th. Spinal cord intramedullary pressure in thoracic kyphotic deformity: a cadaveric study. Spine (Phila Pa 1976) 2012; 37: E224-30.

4. Shimizu K, Nakamura M, Nishikawa Y, Hijikata S, Chiba K, Toyama Y. Spinal kyphosis causes demyelination and neuronal loss in the spinal cord: a new model of kyphotic deformity using juvenile Japanese small game fowls. Spine (Phila Pa 1976) 2005; 30: 2388-92.

5. Cabraja M, Abbushi A, Kroppenstedt S, Woiciechowsky C. Cages with fixation wings versus cages plus plating for cervical reconstruction after corpectomy-is there any difference? Cent Eur Neurosurg 2010; 71: 59-63.

6. Koeppen D, Piepenbrock C, Kroppenstedt S, Čabraja M. The influence of sagittal profile alteration and final lordosis on the clinical outcome of cervical spondylotic myelopathy. A Delta-Omega-analysis. PLoS One 2017; 12: $\mathrm{e} 0174527$.
7. Gercek E, Arlet V, Delisle J, Marchesi D. Subsidence of stand-alone cervical cages in anterior interbody fusion: warning. Eur Spine J 2003; 12: 513-6.

8. Bazaz R, Lee MJ, Yoo JU. Incidence of dysphagia after anterior cervical spine surgery: a prospective study. Spine (Phila Pa 1976) 2002; 27: 2453-8.

9. Stein MI, NayakAN, Gaskins RB3rd, Cabezas AF, Santoni BG, Castellvi AE. Biomechanics of an integrated interbody device versus ACDF anterior locking plate in a single-level cervical spine fusion construct. Spine J 2014; 14: 128-36.

10. Scholz M, Schleicher P, Pabst S, Kandziora F. A zero-profile anchored spacer in multilevel cervical anterior interbody fusion: biomechanical comparison to established fixation techniques. Spine (Phila Pa 1976) 2015 1;40: E375-80.

11. Nemoto O, Kitada A, Naitou S, Tachibana A, Ito Y, Fujikawa A. Stand-alone anchored cage versus cage with plating for single-level anterior cervical discectomy and fusion: a prospective, randomized, controlled study with a 2-year follow-up. Eur J Orthop Surg Traumatol 2015; 25(Suppl 1): S127-34.

12. Barbagallo GM, Romano D, Certo F, Milone P, Albanese V. Zero-P: a new zero-profile cage-plate device for single and multilevel ACDF. A single institution series with four years maximum follow-up and review of the literature on zero-profile devices. Eur Spine J 2013; 22 (Suppl 6): S868-78.

13. Sun Z, Liu Z, Hu W, Yang Y, Xiao X, Wang $\mathrm{X}$. Zero-profile versus cage and plate in anterior cervical discectomy and fusion with a minimum 2 years of follow-up: A meta-analysis. World Neurosurg 2018; 120: e551-61.

14. Song KJ, Yoon SJ, Lee KB. Three- and four-level anterior cervical discectomy and fusion with a PEEK cage and plate construct. Eur Spine J 2012; 21: 2492-7.

15. Xiao SW, Jiang $H$, Yang LJ, Xiao ZM. Anterior cervical discectomy versus corpectomy for multilevel cervical spondylotic myelopathy: a meta-analysis. Eur Spine J 2015; 24: 31-9. 
16. Song KJ, Taghavi CE, Hsu MS, Lee KB, Kim $\mathrm{GH}$, Song JH. Plate augmentation in anterior cervical discectomy and fusion with cage for degenerative cervical spinal disorders. Eur Spine J 2010; 19: 1677-83.

17. Bose B. Anterior cervical fusion using Caspar plating: Analysis of results and review of the literature. Surg Neurol 1998; 49: 25-31.

18. Stewart M, Johnston RA, Stewart I, et al. Swallowing performance following anterior cervical spine surgery. Br J Neurosurg 1995; 9: 605-9.

19. Winslow CP, Winslow TJ, Wax MK. Dysphonia and dysphagia following the anterior approach to the cervical spine. Arch Otolaryngol Head Surgery 2001; 127:51-5.

20. Riley LH 3rd, Skolasky RL, Albert TJ, Vaccaro AR, Heller JG. Dysphagia after anterior cervical decompression and fusion: prevalence and risk factors from a longitudinal cohort study. Spine (Phila Pa 1976) 2005; 30: 2564-9.

21. Fountas KN, Kapsalaki EZ, Nikolakakos LG, SmissonHF,JohnstonKW, GrigorianAA, etal.. Anterior cervical discectomy and fusion associated complications. Spine (Phila Pa 1976) 2007; 32: 2310-7.

22. Di Capua J, Somani S, Kim JS, Phan K, Lee NJ, Kothari P, et al. Elderly age as a risk factor for 30-day postoperative outcomes following elective anterior cervical discectomy and fusion. Global Spine J 2017; 7: 425-31.

23. Omidi KF, Ghayem HE, Ghandehari R. Impact of age and duration of symptoms on surgical outcome of single-level microscopic anterior cervical discectomy and fusion in the patients with cervical spondylotic radiculopathy. Neurosci J 2014; 2014: 808596.
24. Chotai S, Parker SL, Sielatycki JA, Sivaganesan A, Kay HF, Wick JB, et al. Impact of old age on patient-report outcomes and cost utility for anterior cervical discectomy and fusion surgery for degenerative spine disease. Eur Spine J 2017; 26: 1236-45.

25. Aekplakorn W, Mo-Suwan L. Prevalence of obesity in Thailand. Obes Rev 2009; 10: 589-92.

26. Perez-Roman RJ, McCarthy D, Luther EM, Lugo-Pico JG, Leon-Correa R, Gaztanaga W, et al. Effects of body mass index on perioperative outcomes in patients undergoing anterior cervical discectomy and fusion surgery. Neurospine 2021; 18: 79-86.

27. Basques BA, Khan JM, Louie PK, Mormol J, Heidt S, Varthi A, et al. Obesity does not impact clinical outcome but affects cervical sagittal alignment and adjacent segment degeneration in short term follow-up after an anterior cervical decompression and fusion. Spine J 2019; 19: 1146-53.

28. Cabraja M, Oezdemir S, Koeppen D, Kroppenstedt S. Anterior cervical discectomy and fusion: comparison of titanium and polyetheretherketone cages. BMC Musculoskelet Disord 2012; 13: 172.

29. Lee CH, Hyun SJ, Kim MJ, Yeom JS, Kim $\mathrm{WH}$, Kim KJ, et al. Comparative analysis of 3 different construct systems for single-level anterior cervical discectomy and fusion: stand-alone cage, iliac graft plus plate augmentation, and cage plus plating. J Spinal Disord Tech 2013; 26: 112-8.

30. Scholz M, Schnake KJ, Pingel A, Hoffmann R, Kandziora F. A new zero-profile implant for stand-alone anterior cervical interbody fusion. Clin Orthop Relat Res 2011; 469: 666-73. 